\title{
Creating Blockchain Driven Ecosystem Model for Enterprise Financial Management
}

\author{
Quan LIU and Yun-Kui SONG* \\ Guangzhou College of Commerce, Huang Pu District, Guangzhou, Guang Dong, China \\ angelaliu2015@163.com \\ songyk@csg.cn
}

\section{Keywords: blockchain, ecosystem model, financial management, enterprise}

\begin{abstract}
Blockchain technology is broadly used for financial transactions at present in many fields. Technical features of blockchain technology are decentralization, trustworthiness, verifiability, and programmability. Financial management ecosystem is a system model based on natural ecosystems. Financial ecosystem model links the internal and external environment of the enterprise development and the various departments and institutions to contribute to financial management. Financial ecosystem model has a certain role in promoting the financial management level of enterprises. Applying the blockchain to the financial ecosystem is the development trend of blockchain application. The article constructs the financial management ecosystem model based on blockchain and analyzes the problems existing in the blockchain application. Suggestions for improvement to promote the application of blockchain in the financial ecosystem model.
\end{abstract}

\section{Introduction}

Blockchain is an underlying technology in Bitcoin[1]. With the effective containment of Bitcoin in the world, blockchain technology in the underlying technology of Bitcoin has received wide attention from all walks of life and has brought great influence to the global market economy. The establishment of Woolf University in 2018 is the world's first blockchain university while the first blockchain e-wallet cross-border remittance service is lauched, which marks the beginning of the blockchain application in the economic market[6]. Blockchain has the characteristics of decentralization, openness, autonomy, information not tampering, and anonymity which is also the basis for the wide application of blockchain.

Financial management is the foundation of enterprise development which directly affects the survival of enterprises. With the increase in the application of blockchain technology in the financial services, it has also begun to be applied in financial management. Financial ecosystem model combines the theories of financial and ecological, is an ecological manifestation of financial theory practice. In the financial ecosystem model, the internal and external environment of the enterprise development and the various departments are linked, interacting with each other to lay a good foundation for the financial management of the enterprise. The application of blockchain technology in financial management is also beginning to be reflected in the financial management ecosystem model. In order to promote the application level of financial management ecosystem model, this paper tries to creating blockchain driven ecosystem model for enterprise financial management and points out the construction process of financial management ecosystem model, etc. Some analysis and suggestions for improvement are made.

\section{Related Technology}

\subsection{Blockchain Technology}

Blockchain is a distributed database system which integrates many nodes. The core of the system is to realize the operation of the trusted network through decentralization based on the consensus algorithm and encryption technology. In a narrow sense, blockchain is a combination of data arranged in chronological order based on the principle of cryptography[1,2]. The combination has the characteristics of decentralization and non-tamperability. In a broad sense, blockchain technology is based on the distributed consensus algorithm that the decentralized network structure 
of data storage and data code can be realized. The network structure has high data transmission security and uses script to perform data operation command characteristics. Regardless of how the blockchain is understood, it consists mainly two parts, the block and the chain. A block refers to a data block. Data information corresponding to the block is recorded in different blocks, and the data information is recorded in time series and forms a link, which becomes a data chain, thereby forming a current blockchain. Blockchain technology has the following four main characteristics[1]:

(1) Decentralization

Blockchain is a distributed architecture based on nodes that form a protocol network in a peer-to-peer fashion. If the data information on a node is lost or damaged, it will not pose a threat to the entire data security, that is, the data on a single node cannot have a greater impact on the overall data. Based on the fact that the nodes in the block chain do not affect each other, making the blockchain decentralized. In the form of distributed data storage, the blockchain effectively manages a series of processes such as data verification, transmission and management since blockchain-based information system is more resistant to deformation when applied.

(2) De-trust

When data information is transmitted, each node needs to be verified with the help of digital signature technology, and this verification process can be implemented without the need for each node to trust each other. Therefore, the application of digital signature technology makes the blockchain have the characteristics of detrust. When digital signature technology is applied, data information is encrypted and decrypted mainly on the basis of two asymmetric encryption technologies, public key and private key. The public key is completely public, and the authenticity of the data information is guaranteed. The private key is confidential, and the security of data transmission is guaranteed based on this. The existence of the detrust feature makes it possible to achieve the above-mentioned goals only by using an encryption algorithm and a distributed consensus algorithm when data is transmitted between the nodes of the blockchain or when consensus needs to be reached.

(3) Verifiability

Data validation in the blockchain affects data security. Among many algorithms, time stamp technology with data storage verification and information traceability is widely used in blockchain verification. Data tampering and forgery are effectively solved by time stamp technology.

(4) Programmability

The programmability feature means that the blockchain system can be reprogrammed on the basis of the block script code when applied, so that the blockchain system can form an automated and efficient smart contract when applied. Smart contracts are a scenario-triggered procedural rule. Intelligent, automated, and decentralized are their biggest features. It will judge and execute the conditions of the contract execution according to the state rules preset by the system, and the process is intelligent and automated, which highlights the fairness and impartiality of the contract execution, thereby improving effectiveness of contract execution.

\subsection{Financial Management Ecosystem}

The presentation of financial ecosystem is based on natural ecosystems. Natural ecosystem refers to an ecosystem composed of biomes and non-biological natural factors, which directly or indirectly affects human social life. We can learn from the principles of ecology to manage corporate finance, and use financial management as a hub to integrate the internal and external environment of the enterprise and resources to maximize the enterprise value[3,4].

Firstly, financial ecosystem is based on natural ecosystems. Financial management of enterprises and the individual organisms in the natural ecosystem have certain commonalities. Financial ecosystem regards the enterprise financial center as a living organism. The internal and external environment of the enterprise and various organizations are also regarded as living bodies. These living bodies are interrelated and mutually influential, and are not isolated. Therefore, from this perspective, it is very simple to understand the financial ecosystem. That is, there is no independent 
relationship between the enterprise financial center and the internal and external environment of the enterprise and the organizations, but a connection between each other and mutual influence.

Secondly, the proposed financial ecosystem is a new financial management strategy. The reference to the concept of natural ecosystems to look at corporate financial management is an important manifestation of the transformation of corporate financial management concepts, a major change in corporate financial management models, and also for enterprises to adapt to the new environment and new era development requirements. Financial ecosystem has the following four characteristics[3]:

(1) Dynamic

Natural ecosystem is not a constant layer, it is always in dynamic change, and the links between life organisms are always changing. Therefore, financial ecosystem is also dynamic, and the simple and complex relationships between the agencies are constantly changing.

(2) Openness

Natural ecosystems have a certain degree of openness, that is, energy and materials can be exchanged between organisms and the outside world. In the financial ecosystem, there is also a certain amount of energy and material exchange between the various entities. If the external environment of an enterprise changes, the internal environment of the enterprise will also change. Even if the change is small, it also shows that there is a certain degree of openness between the internal and external environments, and they are interconnected and communicate with each other.

(3) Holistic

Natural ecosystem is a large system, and once the organisms are divided into the system, they are no longer independent, but connected with each other to form the integrity of the natural ecosystem. If the organism is isolated, the integrity of the system will also be destroyed. The same is true of the financial ecosystem. After various factors constitute the financial ecosystem, they are not isolated, but show a wholeness, if multiple factors are missing, the holistic character will also not exist.

\section{(4) Cyclical}

Natural ecosystem has been in an infinite loop, so that the organisms in the ecosystem form a complete and stable ecosystem, ensuring the balance and stability of the ecosystem. In the financial ecosystem, various factors and organisms are closely related to the development of the enterprise, and they are interconnected and affect each other, such as human capital and social capital, which are necessary for the development of the enterprise. In order to meet the needs of various organisms, the company needs to allocate resources reasonably. Once the needs of various factors are met, it will also create more value and bring more demand to the company. Therefore, company needs to invest resources again, and iterate cycles to promote its development.

\section{Constructing Financial Management Ecosystem Model Based on Blockchain}

\subsection{Trading Process of Financial Management Ecosystem Based on Blockchain}

Electronic financial management ecosystem uses information technology to support the security and transitivity of electronic financial data to achieve financial data sharing. The main body involved in the electronic financial management system includes government agencies, enterprises, and other taxpayers. The first step to mastering blockchain information flow is to get the transaction flow. Figure. 1 shows the blockchain financial information transaction process: 


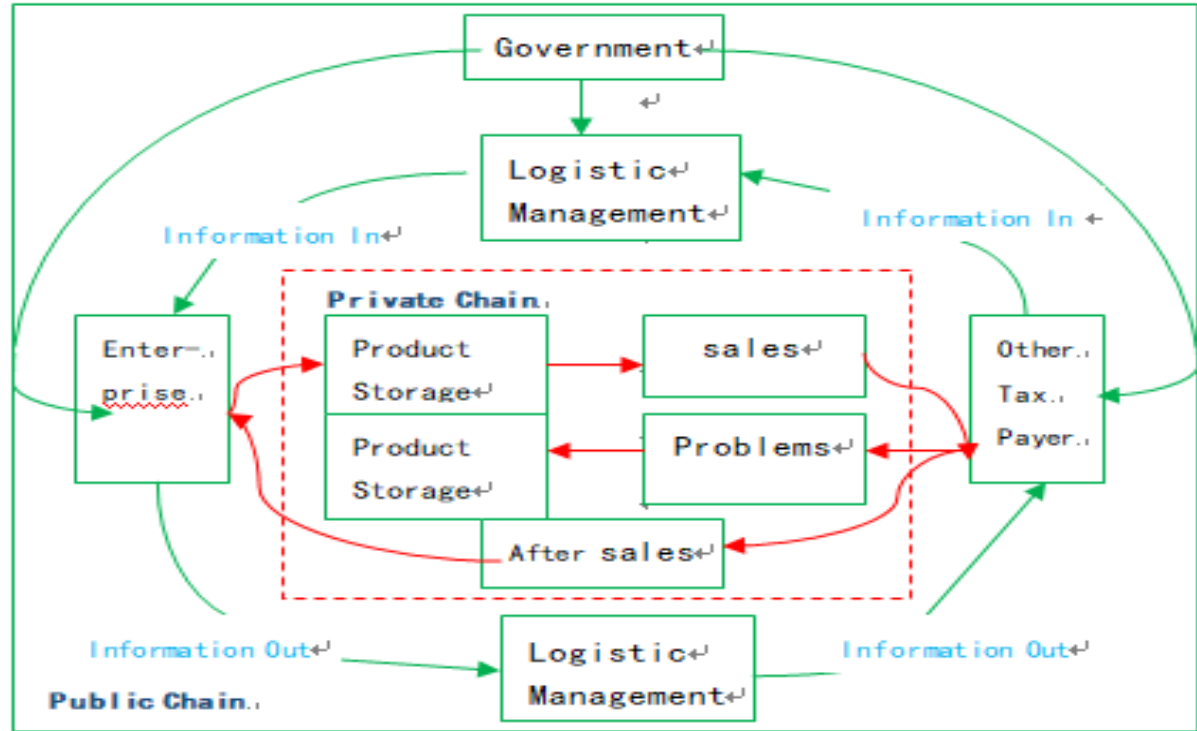

Figure. 1 Blockchain financial information transaction process

Information in financial management is divided into private chains and public chains. Enterprises and other consumer users are merged into the private chain, and strict encryption algorithms are used to protect user information. The private chain contains the business-consumer transaction process. The public chain includes government, enterprises, and logistics. The system uses Hyperledger for transaction bookkeeping, so that transactions can be traced to source. In the blockchain, buyers and sellers automatically match through a contract, and the system automatically clears when the transaction is completed.

\subsection{Auditing Model of Financial Management System Based on Blockchain}

The financial auditing process includes private chain and public chain information. These information are obtained from the blockchain information ecological chain which inchluds: logistics information, product information, personnel information, transaction information, tax information, etc. In the financial management, each node information needs to be transmitted to the network, the system backups data and distributes the data. The matching algorithm is used to verify the information of each node, thereby reducing the data collection work of auditors. At the same time, the transaction process between enterprises is directly stored on the entire network, which improves the audit efficiency. Figure. 2 shows the auditing model of blockchain financial management.

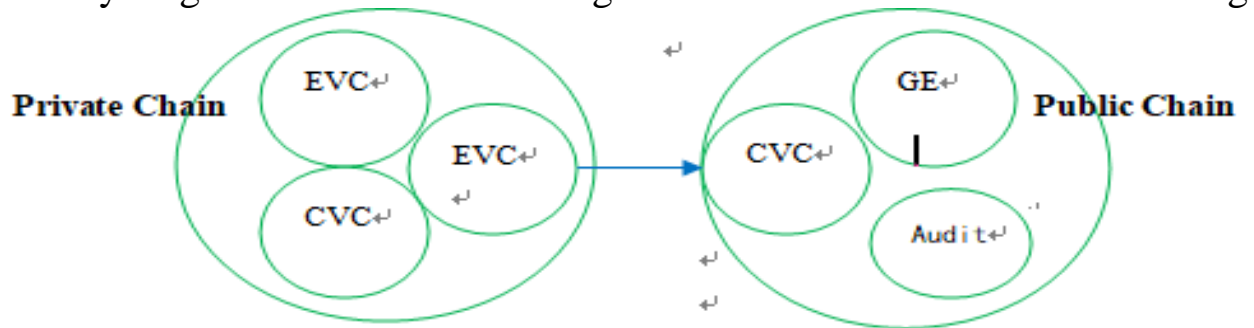

EVC: Enterprise Value Chain; CVC: Customer Value Chain; GE: Govemment Entity

Figure. 2 Blockchain financial management auditing model

With the help of data mining technology, audit efficiency has been improved, and corresponding risks have been identified to facilitate financial auditing.

\subsection{Co-construction of Blockchain Financial Information Ecosystem}

Constructing a good information ecosystem directly affects the information transmission between various information units[5]. The composition of the electronic financial information ecosystem mainly includes government financial management departments, enterprise management departments, and other taxpayers. According to the financial inclusion of users and the relationship between users, the financial ecosystem can be divided into: User Layer, Technology Layer, Intermediary Layer, and Supervision Layer. As shown in Figure. 3: 


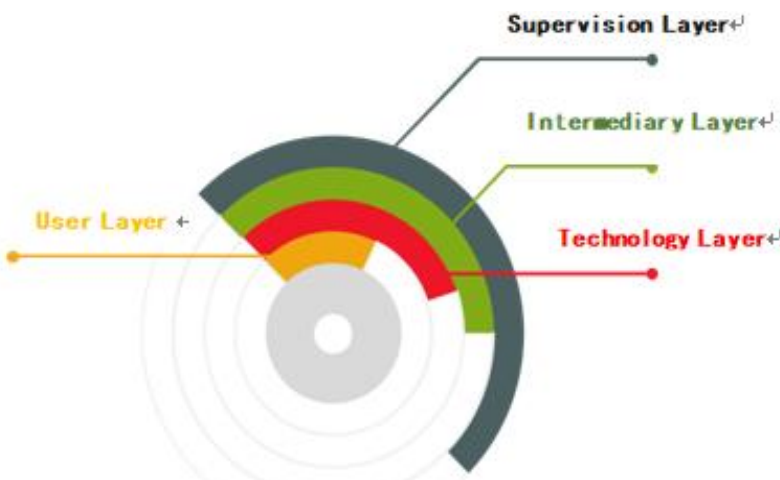

Figure. 3 Electronic Finance Ecosphere

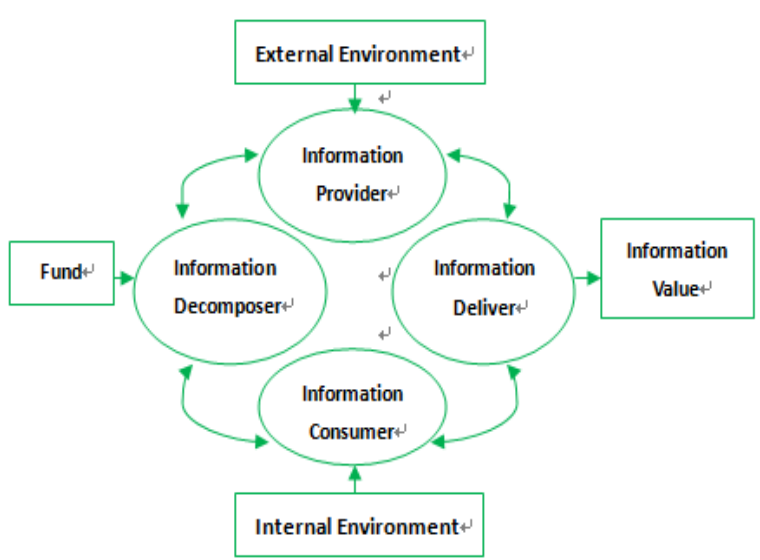

Figure. 4 Electronic financial ecological model

User Layer: enterprise, taxpayer, consumer; Technology Layer: encryption algorithm, recommendation system, mining system, database technology; Intermediary Layer: logistics company, payment company, advertising service company, etc.; Supervision Layer: government agencies, culture, ethics, etc.

\subsection{Blockchain Financial Information Ecosystem Model}

According to the electronic financial information ecological chain and blockchain technology, the constructed financial information ecological model includes financial information data sharing, financial information transparency, financial information authority management, financial information interface data, collaborative management of various financial users, intelligent financial management, etc. This can ensure the safe and stable operation of electronic financial information[5]. As shown in Figure. 4 above.

\section{Recommendations}

\subsection{Financial Data Redundancy Problems and Improvement Countermeasures}

Financial management is mainly realized through the processing of financial data. The increasing financial data is likely to cause data redundancy, which brings certain difficulties to financial management. The decentralization of the blockchain makes it possible to improve data redundancy. That is, related financial information and corporate transaction information data are promoted in each block by the blockchain. The sub-blocks in the database are stored to ensure the accuracy and completeness of each piece of data. The authenticity and accuracy of corporate financial information is directly related to financial security. Enterprises should use the distributed data storage characteristics of the blockchain to store financial information in a distributed state, while improving the configuration level of computer hardware facilities to meet the needs of distributed storage. It realizes the sharing of various financial information and achieves decentralization, thereby ensuring the accuracy and security of financial data.

\subsection{Financial Information Sharing Problems and Improvement Countermeasures}

Enterprise development should pay attention to the utilization and sharing of various information, and incorporate useful information into the enterprise. When the internal information sharing level is high, it means that the internal information of the enterprise is relatively transparent, and each department can get the information they need. At the same time, understanding the overall development strategy of the enterprise is beneficial to the long-term development of the enterprise. It is recommended that enterprises should pay more attention to the sharing of relevant information when using blockchain, so that each department can share accurate information and make reasonable development decisions. There are many types of financial transactions in the enterprise, and various financial information is complicated. If the financial information sharing is realized by the consensus mechanism of the blockchain, the transparency of financial information can be 
effectively improved, and the financial management process can be more clear and transparent, thereby improving the level of financial information management.

\section{Summary}

This paper studies the application of blockchain from the prospective of financial management, and combines blockchain and financial ecological model. As the enterprise financial management work is increasingly complex, it is necessary to integrate the resources and advantages of all aspects of enterprise development on the basis of the application of financial ecosystem, and jointly contribute to financial management. It can be said that the ecosystem model is an effective means for enterprises to conduct financial management, and it is also a development path for future financial management. This paper builds the financial ecosystem model based on the blockchain to promote financial management of the enterprise, enriches the theoretical research of blockchain in financial management to a certain extent.

\section{Acknowledgment}

This research was financially supported by the Guangdong Provincial Department of Education.

\section{References}

[1] Yuan Yong, Wang Feiyue, Blockchain: The state of the art and future trends, J. Acta Automatica Sinica. 42.4 (2016) 3-16.

[2]Xie Hui, Wang Jian, Study on Block Chain Technology and Its Applications, J. Netinfo Security. 9 (2016) 192-195.

[3]Hu Haibo, Zheng Enfeng, Analysis of Corporate Financial Ecosystem and Its Structure, J. Securities \& Futures of China. 11 (2011) 103-104.

[4]Li Yanling, Research on Financial Management Transformation from the Perspective of Ecosystem-Based on the Background of Knowledge Economy Era, J. Communication of Finance and Accounting. 718.26 (2016) 70-72.

[5]Zhang Yanbin, A Model of E-commerce Information Ecosystem Based on Blockchain, J. Research on Library Science. 6 (2018) 33-44.

[6]Radziwill, Nicole, Blockchain Revolution: How the Technology Behind Bitcoin is Changing Money, Business, and the World, J. Quality Management Journal. 25.1 (2018) 64-65. 